\title{
CONTRIBUIÇÃO DA ESCULTURA DENTAL PARA A REALIZAÇÃO DE RESTAURAÇÕES CLÍNICAS E PROTÉTICAS PARA OS ALUNOS DA GRADUAÇÃO DO CURSO DE ODONTOLOGIA DO UNIPÊ.
}

\section{CONTRIBUTION OF DENTAL SCULPTURE FOR CONDUCTING CLINICAL AND PROSTHETIC RESTORATIONS FOR UNDERGRADUATE STUDENTS OF THE SCHOOL OF DENTISTRY UNIPÊ}

\author{
Dayane Franco Barros Mangueira Leite* \\ Iolanda M Cariry L.B. Martins"*** \\ Mariana Trigueiro**** \\ Julianna Larissa de Sousa Santos $\cdots$ \\ Francielly Fernandes Ribeiro de Melo \\ Izabella Araújo Limeira
}

\section{RESUMO}

Introdução: O estudante de Odontologia é capacitado para analisar a forma e a função dos dentes para que posteriormente possa corrigir e restabelecer a fisiologia completa do sistema estomatognático em seus pacientes. O presente trabalho teve como objetivo avaliar as contribuições da escultura dental para confecções de restaurações clínicas e protéticas para os alunos de uma instituição de ensino privado da Paraíba - (UNIPÊ). Métodos: A amostra foi constituída pela totalidade dos alunos a partir do quinto ( $5^{\circ}$ ) período do Curso de Odontologia do Centro Universitário de João Pessoa (UNIPÊ), resultando em 140 estudantes. A coleta de dados foi realizada através de questionário. Os dados foram digitados e analisados através do programa estatístico SPSS versão 13.0. Resultados: A maioria dos participantes era do sexo feminino ( $\mathrm{n}=89,63,6 \%$ ), com média de idade 24,2 $\pm 3,7$ anos. Quanto à contribuição da escultura em cera desenvolvida na disciplina de desenho e escultura dental, 130 (92,9\%) acreditaram no desenvolvimento da habilidade manual. Se a habilidade manual desenvolvida na prática de escultura em cera facilitou a confecção de restaurações em amálgama, 86 (61,4\%) afirmaram positivamente, e em resina, 123 (87,9\%). Conclusões: A escultura dental foi de suma importância para confecção de restaurações clínicas e protéticas; A habilidade manual desenvolvida facilitou a confecção de restaurações tanto em amálgama como em resina; Os alunos que obtiveram maior aproveitamento na disciplina de desenho e escultura consideraram o trabalho de restaurações clínicas e protéticas de mais fácil execução, tendo em vista os conhecimentos adquiridos na disciplina.

Descritores: Escultura • Aptidão • Estética dentária

\section{ABSTRACT}

Introduction: The dental student is able to analyze the form and function of the teeth so that later can correct and restore the complete physiology of the stomatognathic system in his patients. This study aimed to evaluate the contributions of the dental sculpture to clothing dental restorations and prosthetic clinics for students of a private educational institution of Paraíba-(UNIPÊ). Methods: The sample was constituted by all students from the fifth (5th) period of the School of Dentistry of the University Center of João Pessoa (UNIPÊ), resulting in 140 students. Data collection was conducted through a questionnaire. Data were entered and analyzed using SPSS version 13.0. Results: Most participants were female $(n=89,63.6 \%)$ with mean age $24.2 \pm 3.7$ years. Regarding to the contribution of the dental sculpture developed in the discipline of drawing and dental sculpture, 130 $(92.9 \%)$ believed in the development of manual ability. If the manual skill developed in the practice of dental sculpture facilitated the manufacture of amalgam restorations, $86(61.4 \%)$ stated positively, and resin, 123 $(87.9 \%)$. Conclusions: The dental sculpture was of paramount importance for making clinical and prosthetic restorations; The manual ability developed facilitated the manufacture of amalgam restorations as well as in resin; Students who had higher utilization in the discipline of drawing and dental sculpture considered the work of prosthetic and clinic restorations easier to perform, given the knowledge gained in the discipline.

Descriptors: Sculpture $\bullet$ Aptitude $\bullet$ Esthetics, dental

* Instituição em que foi feito o estudo: Centro Universitário de João Pessoa

** Doutora em Estomatologia pela Universidade Federal da Paraíba, Professora da Universidade Federal da Paraíba e Professora de Desenho e Escultura do Centro Universitário de João Pessoa, BR 230, KM 22 e-mail: dayanemangueira@gmail.com

*** Doutoranda em Estomatologia pela Universidade Federal da Paraíba, Professora da Escola Técnica de Saúde da Universidade Federal da Paraíba, Cidade Universitária, João Pessoa-PB, e-mail: iolandacariry@gmail.com

**** Mestre em Ciências Odontológicas pela Faculdade de Odontologia da Universidade de São Paulo, Professora de Desenho e Escultura do Centro Universitário de João Pessoa, BR 230, KM 22, e-mail: marianatrigueiro@hotmail.com

***** Graduanda em Odontologia pelo Centro Universitário de João Pessoa, BR 230, KM 22, e-mail: juliannasousa_@hotmail.com

****** Graduanda em Odontologia pelo Centro Universitário de João Pessoa, BR 230, KM 22, e-mail: francielly.fernandes1 @ hotmail.com

******* Graduada em Odontologia pelo Centro Universitário de João Pessoa, BR 230, KM 22, João Pessoa-PB, e-mail: izabella_cz@hotmail.com 
LEITE DFBM

MARTINS IMCLB

TRIGUEIRO M

SANTOS JLS

MELO FFR

LIMEIRA IA

CONTRIBUIÇÃO

DA ESCULTURA

DENTAL PARA A

REALIZAÇÃO DE

RESTAURAÇÕES

CLINNICAS E

PROTÉTICAS

PARA OS ALUNOS

DA GRADUAÇÃO

DO CURSO DE

ODONTOLOGIA DO

UNIPE

204

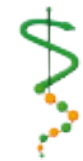

REV, ODONTOL,

UNIV, CID, SÃO

PAULO

2013; 25(3): 203-

7, SET-DEZ

\section{IN TRODUÇÃO}

A disciplina de Escultura Dental proporciona ao aluno de graduação a oportunidade de desenvolver a habilidade manual e de adquirir visão geral do dente, que é fundamental para o trabalho de restauração e reintegração do dente ao aparelho estomatognático ${ }^{1}$. É considerada a área da Odontologia que visa prover a reprodução fiel da forma anatômica dos dentes naturais ${ }^{2}$.

Propostas de ensino de Escultura Dental são desenvolvidas para que o aluno entre em contato com os princípios básicos de oclusão já no início do curso. A escultura, além de trabalhos em cera, desenvolve-se através de técnicas restauradoras atuais como: facetas, incrustações em resina composta, assim como restaurações em amálgama, resina composta entre outras. Com isso, o estudante de Odontologia tem contato com parte clínica e aplicação da escultura e oclusão dental na prática diária, preparando-se para outras disciplinas ${ }^{1,3}$.

A Disciplina Desenho e Escultura Dental desenvolve a habilidade manual dos alunos e seu estudo orienta o desenvolvimento de outros ramos específicos, tais como Dentística e Prótese Dentária. A relevância dos conhecimentos adquiridos na referida disciplina pode ser observada em Dentística, por exemplo, para execução das restaurações dentárias, na qual se exprime a necessidade de conhecer as características de cada estrutura anatômica do elemento dentário. Nota-se sua importância pela necessidade do restabelecimento dos pontos de contato oclusais impedindo que a oclusão fique em desarmonia e traga comprometimento para o aparelho estomatognático, por disfunção nos dentes, no periodonto, na articulação temporomandibular, nos músculos, resultando em sintomatologia dolorosa ${ }^{4}$.

O aluno precisa entender que todo aspecto morfológico estudado tem um significado funcional e deve ser reproduzido na escultura com precisão. Assim, um contorno mal feito, a falta de um sulco, uma crista fora de posição comprometeriam a função ${ }^{5}$.

Com relação à Prótese Dentária, o pre- paro exige que o profissional conheça a morfologia do órgão dental a ser reproduzido. Para se obter uma boa estética e função, deve-se considerar uma tomada correta da dimensão vertical, o plano oclusal protético, linha mediana, linha dos caninos e linha alta do sorriso. Para Gennari Filho et al. ${ }^{6}(2003)$ qualquer tipo de apareIho protético tem o intuito de estabelecer uma proximidade de semelhança com a estrutura biológica perdida, tanto no regimento de suas funções quanto no restabelecimento psicossocial do paciente. $\mathrm{E}$ esse conhecimento é obtido, inicialmente, na graduação de Odontologia na disciplina de Desenho e Escultura Dental, na qual os alunos desenvolvem o seu aprendizado direcionado à escultura e reposição correta dos elementos dentários.

Com base nisso, objetivou-se avaliar as contribuições da disciplina de desenho e escultura dental em cera para a realização de restaurações clínicas e protéticas para os alunos da graduação do Curso de Odontologia do UNIPÊ.

\section{MÉTODOS}

A presente pesquisa foi aprovada pelo Comitê de Ética em Pesquisa do Centro Universitário de João Pessoa - CEP/UNIPÊ, em sua $38^{\circ}$. Reunião Ordinária realizada em 17.05.11, com base na Resolução 196/96 do Conselho Nacional da Saúde/ MS.

A amostra foi constituída pela totalidade dos alunos a partir do quinto $\left(5^{\circ}\right)$ período do Curso de Odontologia do Centro Universitário de João Pessoa (UNIPÊ), resultando em 140 estudantes.

A coleta de dados foi realizada através de questionário, respondido pelos alunos nas dependências da própria instituição de ensino, após a explanação dos objetivos e justificativa do estudo e, em caso de aceite em participar, a assinatura do Termo de Consentimento Livre e Esclarecido. O questionário continha perguntas envolvendo questões tais como: a restauração dental requer conhecimentos anátomo-funcionais; contribuição da escultura dental em cera desenvolvida na disciplina de Desenho e Escultura Dental no desenvolvimento da habilidade manual; se a habilidade manual desenvolvida na prática 
de escultura em cera facilita a confecção de restaurações em amálgama; em resina; na confecção de provisórios; o quanto a prática de escultura em cera é importante para a realização de procedimentos clínicos; o quanto a prática de escultura em cera é importante para a fixação de detalhes anatômicos; a disciplina de escultura dental em cera orientou melhor na construção de uma prótese mais estética, deixando-a mais natural; o conhecimento obtido na disciplina de escultura dental em cera traz mais segurança para a realização de restaurações; se o aluno já teve experiência anterior à disciplina Desenho e Escultura Dental com relação à prática de escultura em cera.

Os dados foram digitados e analisados através do programa estatístico SPSS versão 13.0 .

\section{RESULTADOS}

Dos 140 questionários aplicados nas turmas do $5^{\circ}$ (quinto) ao $9^{\circ}$ (nono) período, o quinto período respondeu a 33 $(23,6 \%)$; o sexto, a $37(26,4 \%)$; o sétimo, a $18(12,9 \%)$; o oitavo, a $28(20,0 \%)$ e o nono, a 24 (17,1\%). Em relação ao sexo, a maioria dos participantes foi do sexo feminino ( $\mathrm{n}=89,63,6 \%)$, com média de idade $24,2 \pm 3,7$ anos.

Quando questionados se a restauração dental requer conhecimentos anátomo-funcionais, 139 (99,3\%) dos participantes acreditaram que sim. Quanto à contribuição da escultura em cera desenvolvida na disciplina de desenho e escultura dental, $130(92,9 \%)$ acreditaram no desenvolvimento da habilidade manual. Se a habilidade manual desenvolvida na prática de escultura em cera facilitou a confecção de restaurações em amálgama, 86 (61,4\%) afirmaram positivamente, e em resina, $123(87,9 \%)$.

Em uma escala de 0 (zero) a 10 (dez) com relação à prática de escultura ser importante para realização de procedimentos clínicos, $31,4 \%$ deram uma importância de 8 (oito). Novamente, em uma escala de 0 (zero) a 10 (dez), quando se questionou sobre a prática de escultura em cera como importante para a fixação de detalhes anatômicos, 30\% responderam 10.
Quanto à confecção de provisórios ser facilitada pela habilidade manual desenvolvida na escultura dental em cera, a questão foi destinada a alunos a partir do $6^{\circ}$ (sexto) período e $108(77,1 \%)$ responderam a essa questão e a maioria $(n=85$, $60,7 \%$ ) acreditou que a habilidade manual desenvolvida na disciplina de desenho e escultura apoia a confecção de provisório. Também a partir do $6^{\circ}$ (sexto) período, foi perguntado se o aluno acreditava que a disciplina de escultura dental em cera orientou na construção de prótese mais estética deixando-a mais natural, os alunos responderam que sim, pois a partir desta disciplina eles tinham um melhor contato com a real anatomia dos elementos dentários. Quando perguntados se o conhecimento adquirido na disciplina de desenho e escultura dental trouxe mais segurança na realização de restaurações, os alunos afirmaram que sim, por permitir um maior conhecimento em relação à anatomia dos elementos dentais.

Por fim, questionou-se se o aluno já havia tido experiência anterior à disciplina de desenho e escultura dental, com relação à prática de escultura em cera, e 129 $(92,1 \%)$ asseguraram que não.

\section{I SCUSSÃO}

Acredita-se que a habilidade manual é natural aos indivíduos, porém sabe-se que ela pode ser desenvolvida ou melhorada através de boas orientações e intensivos treinamentos ${ }^{7}$. Um estudante de Odontologia deve desenvolver uma percepção estética e ser capaz de analisar a forma e função dos dentes para que possa corrigir e restabelecer a fisiologia completa em seus pacientes ${ }^{1}$.

Com relação ao aprendizado oferecido pelo método em cera, uma das principais observações é a melhora considerável das esculturas apresentadas pelos alunos em trabalhos utilizando resina composta. A maioria dos alunos relatou apresentar meIhor habilidade manual, visto que bons trabalhos em um curto espaço de tempo foram executados, além do que a escultura dental ensinou detalhes, como, por exemplo, proporção entre altura e largura, altura de bossas vestibular e lingual, alinhamento, inclinação e convergência das
LEITE DFBM

MARTINS IMCLB

TRIGUEIRO M

SANTOS JLS

MELO FFR

LIMEIRA IA

CONTRIBUIÇÃO

DA ESCULTURA

DENTAL PARA A

REALIZAÇÃO DE

RESTAURAÇÕES

CLÍNICAS E

PROTÉTICAS

PARA OS ALUNOS

DA GRADUAÇÃO

DO CURSO DE

ODONTOLOGIA DO UNIPE
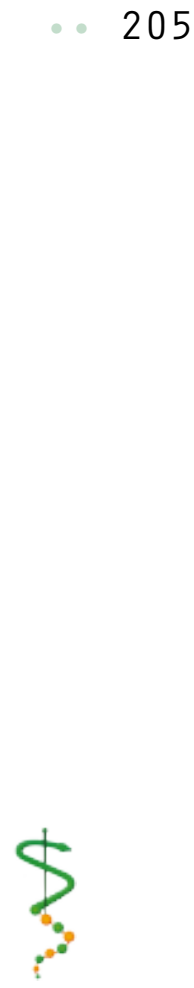

REV. ODONTOL.

Univ, CID. São

PAULO

2013; 25(3): 203-

7, SET-DEZ 
LEITE DFBM

MARTINS IMCLB

TRIGUEIRO M

SANTOS JLS

MELO FFR

LIMEIRA IA

CONTRIBUIÇÃO

DA ESCULTURA

DENTAL PARA A

REALIZAÇÃO DE

RESTAURAÇÕES

CLINNICAS E

PROTÉTICAS

PARA OS ALUNOS

DA GRADUAÇÃO

DO CURSO DE

ODONTOLOGIA DO

UNIPE

\section{6}

ISSN 1983-5183

faces vestibular e lingual, sendo capaz de melhorar a qualidade das esculturas.

Para Santos Júnior ${ }^{8}$ (2000), com o treinamento manual e a percepção de detaIhes anatômicos dos dentes, que é exigida dos alunos, melhora-se a percepção de proporção entre esses elementos.

Inúmeras são as formas e técnicas utilizadas por profissionais da área da Odontologia para restabelecer a anatomia perdida dos dentes. Recuperar a tão valorizada estética do sorriso compreende diversos procedimentos que necessitam, além de conhecimentos técnico-científicos, senso artístico, destreza e habilidade manual, a fim de tornar as restaurações com aspecto mais naturais possíveis 9 .

Para que uma restauração odontológica tenha sucesso, é necessário que ela restabeleça as funções do sistema estomatognático; para isso, além do conhecimento anatômico individual de cada elemento dental, é importante conhecer as relações interproximais, dentro de uma mesma arcada, e relações oclusais, entre dentes de arcos antagonistas ${ }^{10}$. O aluno, ao cursar a disciplina de Escultura, estará apto a não só desenvolver sua habilidade manual, como também a adquirir visão geral do dente, o que facilitará seu trabalho de restauração e integração do dente ao sistema estomatognático ${ }^{11}$.

Considerando a prótese, faz-se necessário manter os traços faciais típicos, o perfil, a forma e suporte dos lábios e a harmonia ao sorrir, para se obter uma fisionomia agradável do paciente. Variações nas anatomias ósseas e mucosas, alterações no relacionamento oclusal, na articulação temporomandibular, perda de dimensão vertical de oclusão e a expectativa do paciente constituem uma combinação de variáveis que necessitam de uma abordagem multidisciplinar ${ }^{9}$.

Vê-se que os conhecimentos e treinamentos conquistados na disciplina, através do método em cera, aprontam o aluno para um desempenho profissional mais consciente e hábil, restabelecendo forma e função dos dentes, obtendo uma boa fisiologia mastigatória, harmonia e estética entre os arcos.

\section{CONCLUSÕES}

- A escultura dental em cera foi de suma importância para confecção de restaurações clínicas e protéticas;

- A habilidade manual desenvolvida na prática de escultura em cera facilitou a confecção de restaurações tanto em amálgama como em resina;

- Os alunos que obtiveram maior aproveitamento na disciplina de desenho e escultura consideraram o trabalho de restaurações clínicas e protéticas de mais fácil execução, tendo-se em vista os conhecimentos adquiridos na disciplina.

\footnotetext{
REV, ODONTOL

UNIV. CID. SÃO PAULO
} 2013; 25(3): 2037 , SET-DEZ : 
LEITE DFBM

MARTINS IMCLB

TRIGUEIRO M

SANTOS JLS

MELO FFR

LIMEIRA IA cácia do método geométrico no aprendizado da escultura dental no curso de graduação em Odontologia. Revista da ABENO 2007 mai.-ago; 7(2):112-6.

2. Vieira G, Caroli A, Garofalo J, Matson E. Escultura dental com auxílio do método geométrico. 4. ed. Ribeirão Preto: Ad-Tech Comunicação; 2003.

3. Vieira D, Carreira A, Ferreira A. Escultura dental: uma nova proposta de ensino. Rev Paul Odontol 1996 mar-abr;18(2):35-9.

4. Costa A, Moura C, Cavalcante G. Ceroplastia em dentes articulados no manequim. In: Costa $A$, editor. Anatomia e Escultura Dental. João Pessoa: Universitária; 2007.

5. Madeira M. Anatomia do dente. 4. ed. São Paulo: Sarvier; 2005.
CONTRIBUIÇÃO DA ESCULTURA DENTAL PARA A REALIZAÇÃO DE RESTAURAÇÕES CLINICAS E PROTÉTICAS PARA OS ALUNOS DA GRADUAÇÃO DO CURSO DE ODONTOLOGIA DO UN I PE zari , Assunção W, Shibayama R.

6. Gennari Filho H, Vedovatto E, LaAvaliação comparativa da posição relativa dos dentes artificiais entre três métodos de inclusão de próteses totais polimerizadas em banho de água quente. Cienc Odontol Bras 2003 out-dez;6(4):32-40.

7. Cantisano W, Palhares R, Santos S. Anatomia dental e sscultura. 3. ed. Rio de Janeiro: Guanabara Koogan; 1987.

8. Santos Júnior J, Fichman D. Escultura e modelagem dental. 6. ed. São Paulo: Santos; 2000.

9 , Rufenacht C. Fundamentos de estética. São Paulo: Quintessence; 1998.

10. Guimarães R, Reis R. Reconstrução da morfologia oclusal através da técnica da matriz individual de acrílico - relato de caso clínico. JBD 2004 abr-jun;3(10):154-9.

11. Della Serra O. Anatomia dental. 2. ed. Rio de Janeiro: Científica; 1995.

Recebido em: 17/09/2013

Aceito em: 02/04/2014

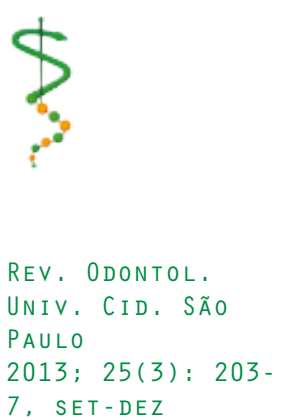

